Research Paper: Gerotarget (Focus on Aging)

\title{
Sub-acute systemic erythropoietin administration reduces ischemic brain injury in an age-dependent manner
}

\author{
Peter Thériault ${ }^{1}$, Audrey Le Béhot ${ }^{1}$, Ayman EIAli' ${ }^{2}$ and Serge Rivest ${ }^{1}$ \\ ${ }^{1}$ Neuroscience Laboratory, CHU de Québec Research Center and Department of Molecular Medicine, Faculty of Medicine, \\ Laval University, Québec City, QC, Canada \\ ${ }^{2}$ Neuroscience Laboratory, CHU de Québec Research Center and Department of Psychiatry and Neuroscience, Faculty of \\ Medicine, Laval University, Québec City, QC, Canada \\ Correspondence to: Serge Rivest, email: serge.rivest@crchudequebec.ulaval.ca
}

Ayman ElAli, email: ayman.el-ali@crchudequebec.ulaval.ca

Keywords: ischemic stroke, erythropoietin, aging, inflammation, monocyte chemotactic protein-1, Gerotarget

Received: March 23, 2016

Accepted: May 19, 2016

Published: May 29, 2016

\section{ABSTRACT}

Stroke is associated with neuroinflammation, neuronal loss and blood-brain barrier (BBB) breakdown. Thus far, recombinant tissue-type plasminogen activator (rtPA), the only approved treatment for acute ischemic stroke, increases the risk of intracerebral hemorrhage and is poorly efficient in disaggregating platelet-rich thrombi. Therefore, the development of safer and more efficient therapies is highly awaited. Encouraging neuroprotective effects were reported in mouse models of ischemic stroke following administration of erythropoietin (EPO). However, previous preclinical studies did not investigate the effects of EPO in focal ischemic stroke induced by a platelet-rich thrombus and did not consider the implication of age. Here, we performed middle cerebral artery occlusion by inducing platelet-rich thrombus formation in chimeric 5- (i.e. young) and 20- (i.e. aged) months old C57BL/6 mice, in which hematopoietic stem cells carried the green fluorescent protein (GFP)-tag. Recombinant human EPO (rhEPO) was administered 24 hours post-occlusion and blood-circulating monocyte populations were studied by flow cytometry 3 hours postrhEPO administration. Twenty-four hours following rhEPO treatment, neuronal loss and BBB integrity were assessed by quantification of Fluoro-Jade B (FJB)-positive cells and extravasated serum immunoglobulins G (IgG), respectively. Neuroinflammation was determined by quantifying infiltration of GFP-positive bone marrow-derived cells (BMDC) and recruitment of microglial cells into brain parenchyma, along with monocyte chemotactic protein-1 (MCP-1) brain protein levels. Here, rhEPO antiinflammatory properties rescued ischemic injury by reducing neuronal loss and BBB breakdown in young animals, but not in aged littermates. Such age-dependent effects of rhEPO must therefore be taken into consideration in future studies aiming to develop new therapies for ischemic stroke.

\section{INTRODUCTION}

Stroke is the second cause of mortality worldwide after heart diseases and occurs following the interruption of blood circulation in brain by cerebral vessel burst (i.e. hemorrhagic stroke, $13 \%$ of cases) or occlusion (i.e. ischemic stroke, $87 \%$ of cases) [1] following an embolus or a local thrombus formation [2]. Thrombosis depends on local platelet aggregation and activation of coagulation factors, leading to the formation of a fibrin-rich clot [3]. Under physiological conditions, a fibrinolytic process is triggered in order to prevent the progression of fibrin thrombi, which is regulated by the interaction between endogenous tissue-type plasminogen activator (tPA), its inhibitors and the plasminogen/plasmin system [4, 5]. Accordingly, the administration of recombinant tPA $\left(\right.$ rtPA, Actilyse ${ }^{\circledR}$ ) has been intensively studied both in animal models and patients $[6,7]$. As vessel recanalization 
induced by rtPA administration improves functional outcome and reduces neurological deficits, it remains the sole acute ischemic stroke treatment [8]. Nonetheless, rtPA presents some concerning limitations such as poor efficiency in disaggregating platelet-rich thrombi $[9,10]$, increased risk of intracerebral hemorrhage [8] and tight administration time-window (i.e. 4.5 hours) [11, 12]. Altogether, these factors restrain its application as less than $10 \%$ of ischemic stroke patients are thrombolysed [13]. Therefore, in order to restore vessel patency after ischemic stroke and improve functional outcomes, it is imperative to develop safer and more efficient therapeutic strategies.

Erythropoietin (EPO), a member of the hematopoietic cytokine superfamily, modulates hematopoiesis and stimulates erythropoiesis in the bone marrow (BM) [14, 15]. During metabolic stress, EPO is produced locally in different organs, such as the brain [16], and acts as a multifunctional protective molecule [17, 18]. Therefore, it was speculated that EPO administration might constitute a promising therapeutic approach in brain disorders, such as ischemic stroke [19]. Indeed, a number of findings demonstrated in vivo robust neuroprotective properties of exogenous EPO [20]. More precisely, systemic EPO administration has been shown to reach the ischemic brain, activating anti-apoptotic and antiinflammatory signaling in neurons and glial cells [20], thus reducing cerebral damage $[21,22]$. As such, this suggests acute and chronic actions for EPO in the ischemic brain.

Although encouraging results were reported, the effects of EPO seem to depend on the time and the animal models of stroke [23]. First, despite age is a major contributor in the prevalence, incidence and outcome of ischemic stroke [24], most in vivo studies were performed in young animals (i.e. 2- to -6-months old). Second, EPO administration is mainly performed before arterial obstruction [25] or at time of reperfusion [26], contrasting with thrombolysis conditions observed in patients. Finally, arterial occlusion in animal models is widely induced by an intraluminal filament or electrocoagulation, whereas in patients, occlusion is due to thrombus formation by embolism or local occlusive thrombosis [2].

Our study is based on the urge of developing new therapeutical approaches that consider age in ischemic stroke models that are more closely associated to the human pathophysiology. Here, we observed neuroprotective effects following sub-acute recombinant human EPO (rhEPO) administration in an ischemic stroke model based on platelet-rich thrombus formation [27], using chimeric 5- (i.e. young) and 20- (i.e. aged) months old mice.

\section{RESULTS}

\section{rhEPO administration limits neuronal loss and BBB breakdown in young animals, but not in aged ones}

In order to evaluate the impact of sub-acute rhEPO administration on neuronal loss following ischemic injury, we quantified FJB-positive neuronal cells by stereological analysis in brains of 5- (i.e. young) and 20- (i.e. aged) months old mice. We observed a significant reduction of FJB-positive cells coverage (Figure 1A, left) and density (Figure 1A, right) in brains of rhEPO-treated young animals in comparison to saline-treated ones, while no changes were observed neither in FJB-positive cells coverage (Figure 1B, left) or density (Figure 1B, right) in aged littermates. Moreover, in order to assess BBB integrity, we measured serum IgG extravasation. We observed that rhEPO significantly reduces IgG extravasation in brains of young animals in comparison to saline-treated ones (Figure 1C). However, no changes were observed in aged littermates (Figure 1D). These results suggest that rhEPO limits neuronal loss and $\mathrm{BBB}$ breakdown in young animals, while no effects were observed in aged ones.

\section{rhEPO administration does not affect blood- circulating monocyte populations}

As mentioned, EPO is a hematopoietic cytokine that can modulate hematopoiesis [15], which can affect the production of bone marrow-derived cells (BMDC) such as monocytes. Therefore, 3 hours post-injection of rhEPO, we assessed by FACS analysis blood-circulating monocyte $(\mathrm{CD} 45+\mathrm{CD} 11 \mathrm{~b}+\mathrm{CD} 115+)$ frequencies, including proinflammatory (Ly6C $\mathrm{C}^{\text {High }}$ ) and patrolling (Ly6C $\left.{ }^{\text {Low }}\right)$ subsets [28], in freshly isolated blood samples from young and aged mice (Figure 2A). Interestingly, we did not observed any changes in frequencies of neither total monocyte nor $\mathrm{Ly} 6 \mathrm{C}^{\mathrm{High}}$ and $\mathrm{Ly} 6 \mathrm{C}^{\mathrm{Low}}$ subsets following rhEPO administration, when compared to saline-treated ones, in both young and aged mice (Figure $2 \mathrm{~B}, 2 \mathrm{C}$ respectively). These results indicate that systemic rhEPO administration does not affect blood-circulating monocyte population frequencies, regardless of age.

\section{rhEPO administration limits BMDC infiltration in the brain parenchyma of young animals, while it remains unchanged in aged littermates}

Chimeric mice $(\mathrm{GFP} \rightarrow \mathrm{WT})$ were generated in order to assess rhEPO's anti-inflammatory effects on BMDC brain infiltration (Figure 1, timeline). Forty-six days post- 
transplantation, we determined chimerism by FACS in freshly isolated blood samples from young (Figure 3A) and aged chimeric mice (Figure 3C). Thereafter, we assessed brain infiltration of GFP+ BMDC via quantification of endogenous monocyte-like GFP+ cells in brains of young (Figure 3B) and aged mice (Figure 3D). We observed a significant reduction of GFP-positive cells coverage area (Figure 3B, left) and density (Figure 3B, right) in brains

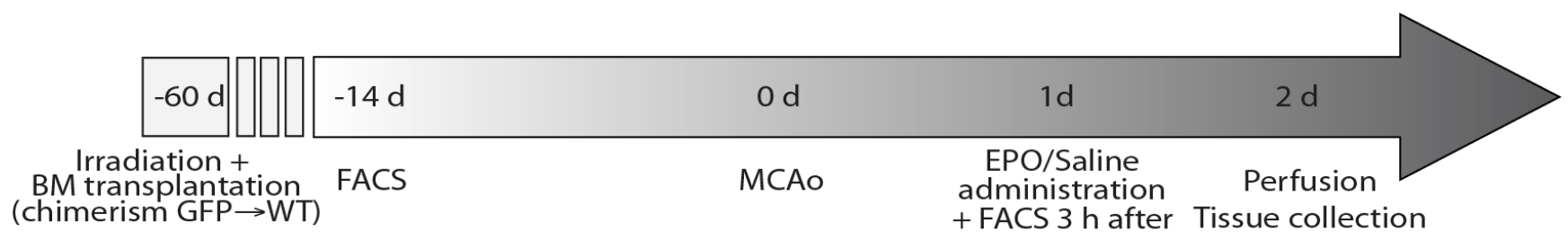

\section{5 months}

A
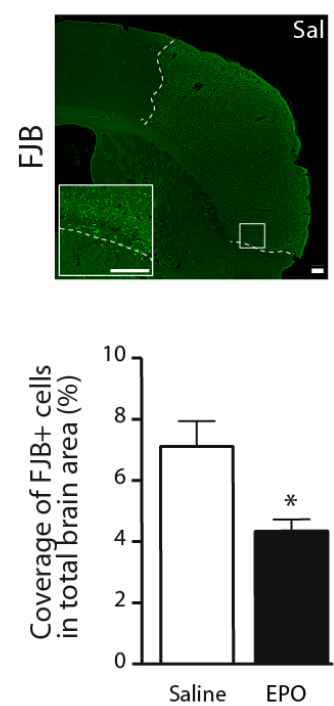

C

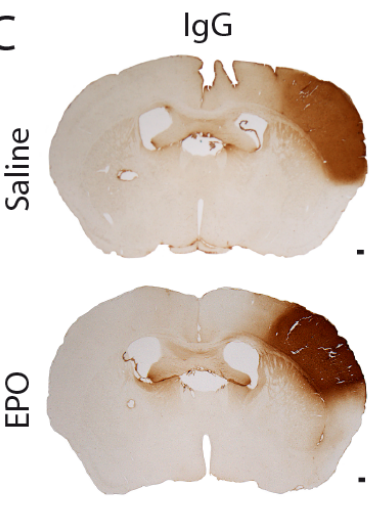

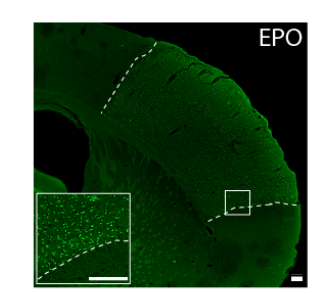
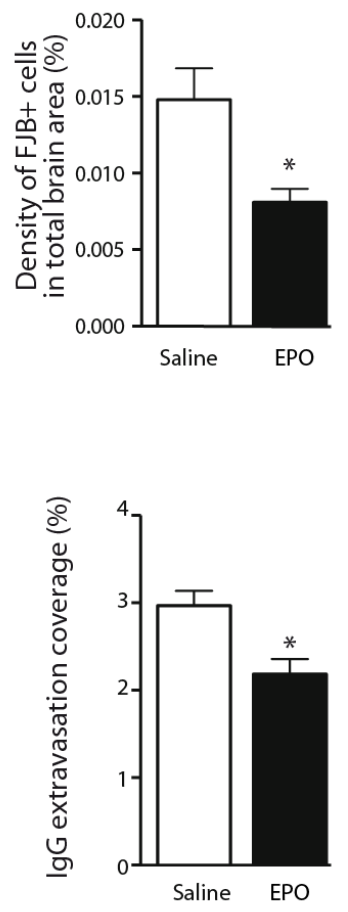

\section{0 months}

B
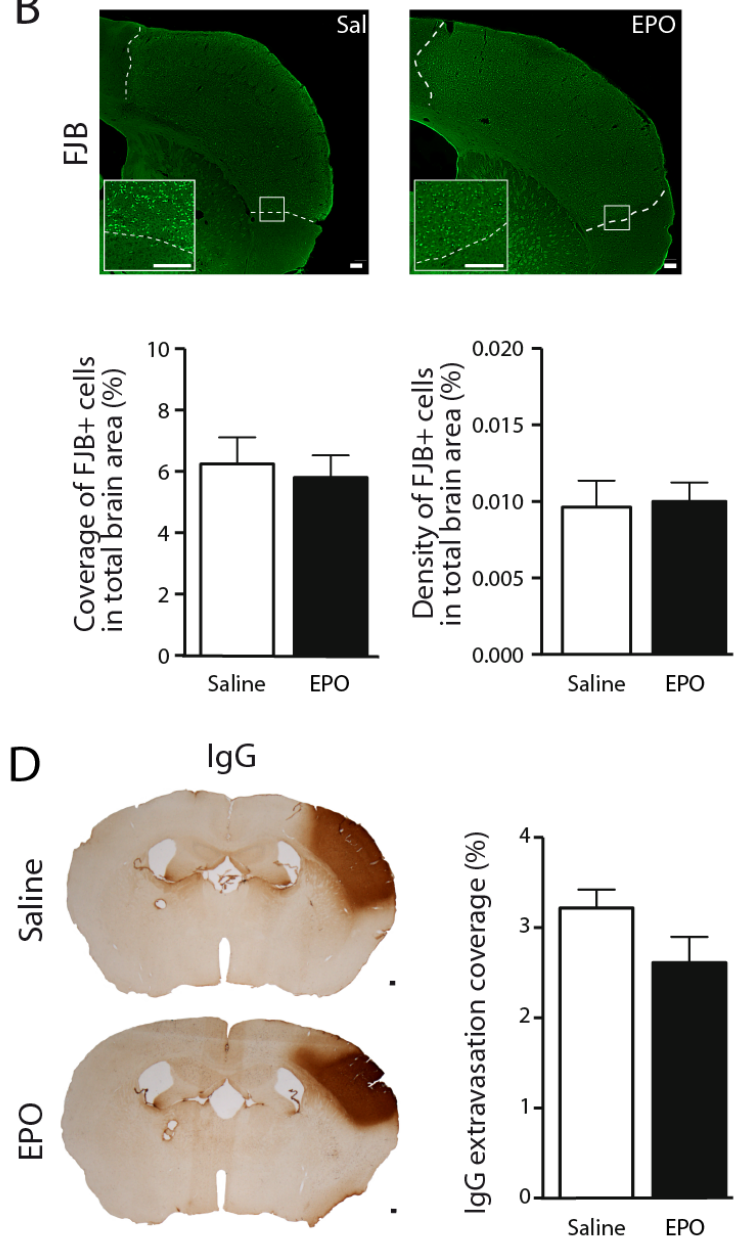

Figure 1: rhEPO administration limits neuronal loss and BBB breakdown in ischemic injury. Timeline of experimentation (grey arrow). Representative images of FJB staining and respective stereological quantifications of FJB-positive cells coverage and density (relative \% of the total brain area) of saline- or EPO-treated 5- (i.e. young) A. and 20- (i.e. aged) B. months old animals. Representative images of IgG staining and respective stereological quantification of IgG extravasation coverage area (relative \% of the total brain area) of saline- or EPO-treated 5- C. and 20- D. months old animals. Values are expressed as means \pm SEM. Statistical analyses were performed using an unpaired $t$-test. ${ }^{*}=p<0.05$ significant difference compared to saline. $\mathrm{n}=8-9$ mice per group. Scale bars $(\mathrm{A}-\mathrm{D})=150 \mu \mathrm{m}$. Abbreviations: BBB: blood-brain barrier; BM: bone marrow; d: day; EPO: erythropoietin; FACS: flow cytometry; FJB: Fluoro-Jade B; GFP: green fluorescent protein; h: hour; IgG: immunoglobulins G; MCAo: middle cerebral artery occlusion; Sal: saline; WT: wild type. 

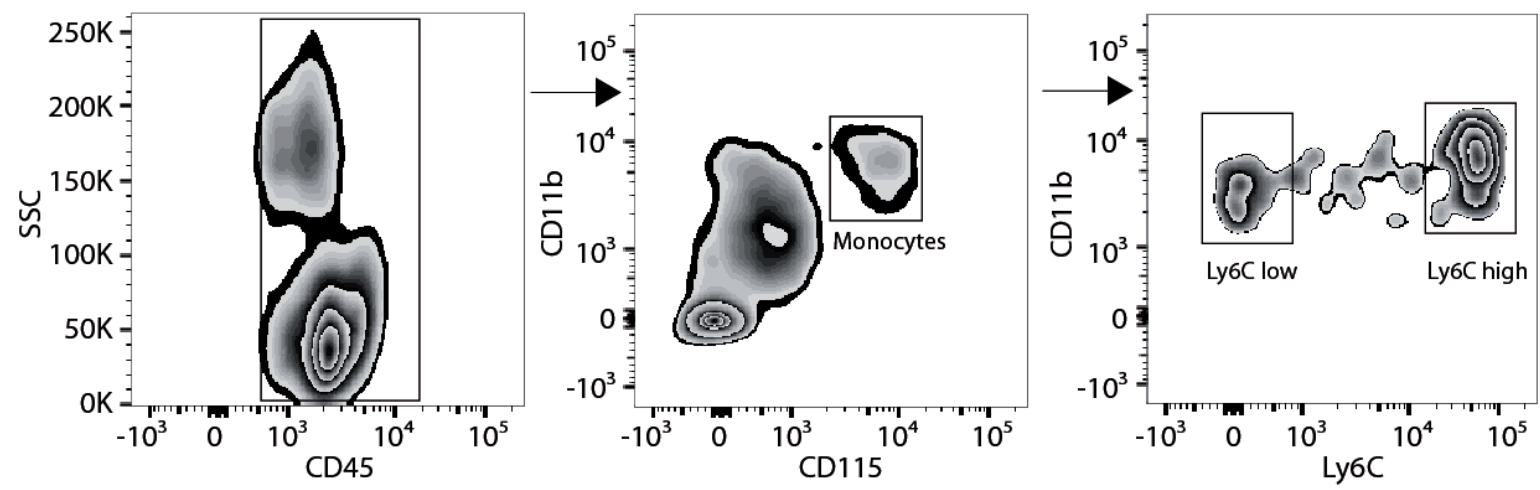

B
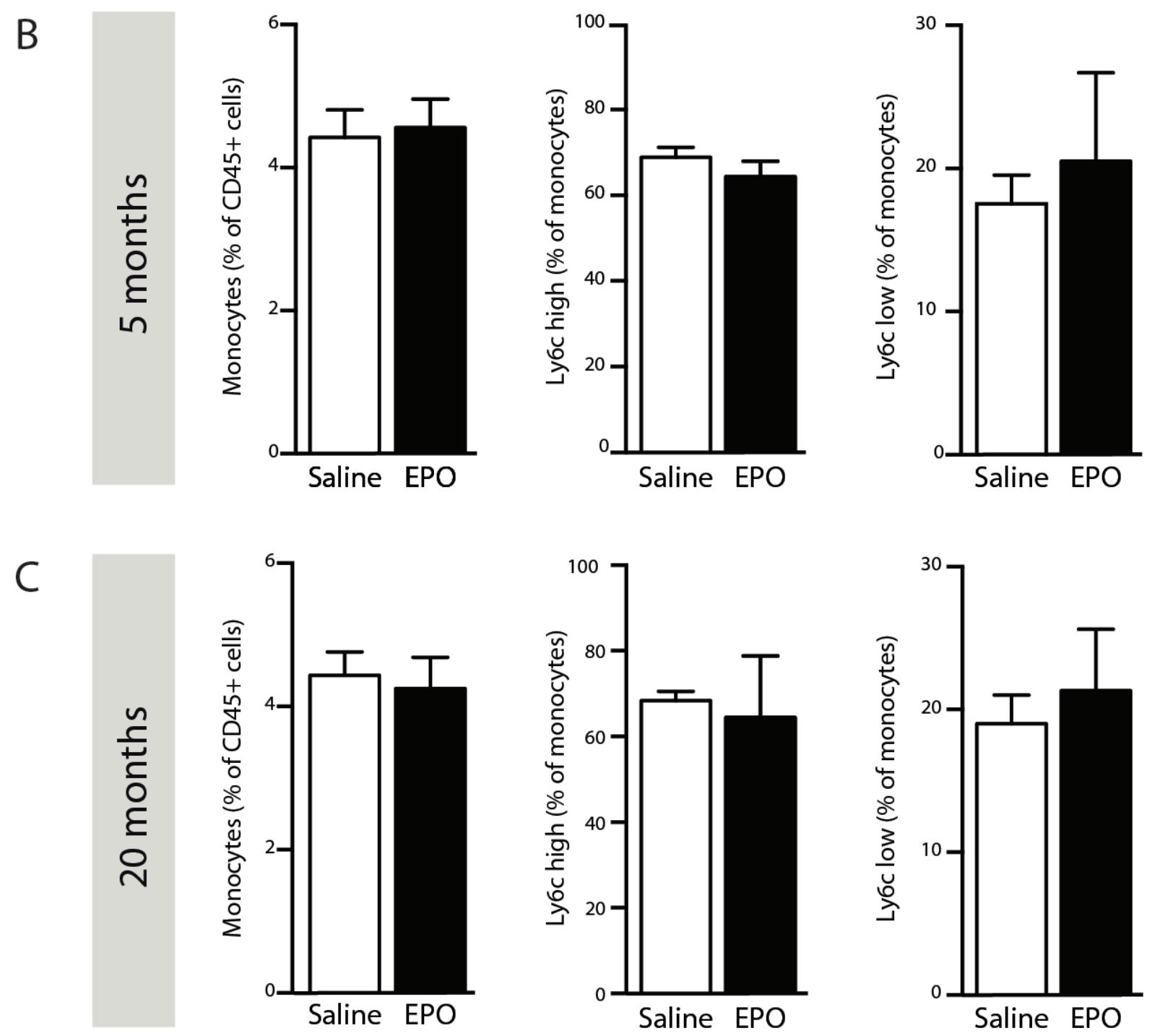

Figure 2: rhEPO administration does not affect blood-circulating monocyte populations. Gating strategy A. to study populations of blood-circulating monocytes; leukocytes (CD45+, left panel), total monocytes (CD45+/CD11b+/CD115+, middle panel), pro-inflammatory (Ly6 $\mathrm{C}^{\text {high }}$ ) and patrolling (Ly6C $\mathrm{C}^{\mathrm{low}}$ ) monocytes (right panel). B., C. Frequencies of total monocytes (relative $\%$ of CD45positive cells; left graph), Ly6C high subset (relative $\%$ of total monocytes; middle graph) and Ly6C $\mathrm{C}^{\text {low }}$ subset (relative $\%$ of total monocytes; right graph) in saline- or EPO-treated 5- (B) and 20- (C) months old animals. Values are expressed as means \pm SEM. Statistical analyses were performed using an unpaired $t$-test. $n=8-9$ mice per group. Abbreviations: EPO: erythropoietin; Sal: saline. 
of rhEPO-treated young mice in comparison to salinetreated ones. However, when comparing the two groups, no changes were detected in aged mice (Figure 3D). Thus, rhEPO administration likely limits the entry of BMDC in the brain parenchyma of young animals, while no changes were observed in aged littermates.

\section{rhEPO administration reduces microglial cells recruitment and monocyte chemotactic protein-1 (MCP-1) protein levels in the ischemic brain of young animals, without any changes in aged ones}

Next, we evaluated the effects of rhEPO systemic administration on microglial cells response following ischemic injury. As such, we quantified IBA-1-positive cells by stereological analysis in brains of young (Figure 4A) and aged mice (Figure 4C). Interestingly, we observed a reduction, although statistically not significant ( $p=$ 0.0583 ), of IBA-1-positive cells density in brains of rhEPO-treated young mice in comparison to saline-treated littermates (Figure 4A, right). However, no changes were found either in rhEPO- or saline-treated aged mice (Figure 4C, right). Additionally, in order to elucidate the mechanism involved in the reduction of BMDC brain infiltration following rhEPO treatment, we investigated MCP-1 (also named chemokine (C-C motif) ligand 2 or CCL2) production, which is a chemokine involved in BMDC (e.g. monocytes) mobilization and recruitment [29]. Therefore, we measured MCP-1 protein levels by western blot analyses in brains of young (Figure 4B) and aged mice (Figure 4D). We detected a significant reduction of $\mathrm{MCP}-1$ protein levels in the ipsilateral hemisphere (i.e. ischemic injury) of rhEPO-treated young mice in comparison to saline-treated ones, while no changes were observed in the contralateral hemisphere (i.e. healthy tissue) of neither rhEPO-treated nor salinetreated mice (Figure 4B). Moreover, no changes were observed in the ipsilateral or contralateral hemispheres in aged mice (Figure 4D). Altogether, these results suggest that rhEPO administration limits microglial cell activation and recruitment in ischemic injury, which consequently reduces MCP-1 production, thus preventing BMDC infiltration in the ischemic brain of young animals, which is not observed in aged animals.

\section{DISCUSSION}

In this study, we evaluated the impact of sub-acute systemic rhEPO administration following ischemic stroke induced by a platelet-rich thrombus, in 5- (i.e. young) and 20- (i.e. aged) months old animals. Our results indicate that rhEPO administration reduces ischemic injury through its anti-inflammatory properties, limiting neuronal loss and $\mathrm{BBB}$ breakdown in an age-dependent manner. More precisely, in young animals, rhEPO significantly attenuates pro-inflammatory response by limiting brain infiltration of $\mathrm{BMDC}$, which is accompanied by a reduced recruitment of microglial cells and decreased MCP-1 productionin the ischemic brain. However, these beneficial effects were not observed in aged animals, suggesting that age-related factors could abolish responsiveness to rhEPO.

In this regard, the majority of focal ischemic stroke models are based on young animals, although age profoundly affects several parameters, including arterial remodeling [30], BBB integrity, glial cells activation, neurogenesis and apoptosis [31, 32], which increase the susceptibility to brain injury $[4,31]$. Therefore, large artery intracranial occlusive disease is considered as the most common subtype of ischemic stroke worldwide [2]. In this pathology, intracranial atherosclerotic lesions are responsible for both artery-to-artery embolisms and in situ occlusive thrombosis, leading to in situ formation of platelet-rich thrombi [2]. These findings underline an important role of age in stroke pathogenesis and thereby, the development of therapeutic strategies.

As such, besides its lack of efficiency for treatment of patients presenting platelet-rich thrombi [9], rtPA treatment increases the risk of intracerebral hemorrhage and presents potential neurotoxic effects, which greatly limit its clinical usage [7]. Therefore, we investigated a novel therapeutic approach based on EPO's cytoprotective effects [20], using an ischemic stroke model induced by platelet-rich thrombus [27]. As mentioned, we reported reduced neuronal loss and $\mathrm{BBB}$ breakdown in young rhEPO-treated animals in comparison to saline-treated littermates, 24 hours post-occlusion. In previous in vivo studies with ischemic mice, EPO was administered at earlier time points. For instance, Wang et al. showed that when administered 2 hours after arterial occlusion (i.e. at beginning of reperfusion), EPO reduces the infarct volume, oedema, BBB leakage and neurologic deficits as soon as 24 hours post-administration [26]. When injected before ischemic stroke [25] or during reperfusion [33], effectiveness of EPO reveals a larger therapeutic timewindow when compared to rtPA, thus suggesting that rhEPO administration should be an effective treatment with a large time window following ischemic stroke, at least in young patients. As such, when administered in patients within 8 hours following ischemic stroke and readministered 24 and 48 hours later, rhEPO is associated with an improvement in the clinical outcome one month after-treatment [34]. In addition, another clinical study reported a significant improvement of the long-term (i.e. 5 years) neurological outcomes of EPO-treated patients, using two consecutive doses of EPO (i.e. 5,000 IU/ dose) administered at 48 hours and 72 hours following ischemic stroke [35], thus suggesting that EPO is an effective treatment for acute ischemic stroke. However, it has been reported that EPO exacerbates cerebral oedema, BBB leakage, and extracellular matrix breakdown in thrombolysed mice [36], suggesting that ischemic stroke 


\section{5 months}

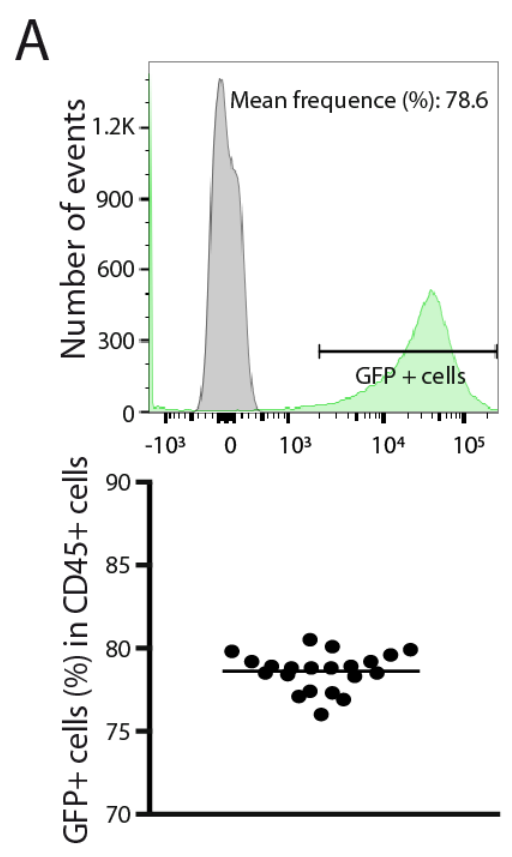

B
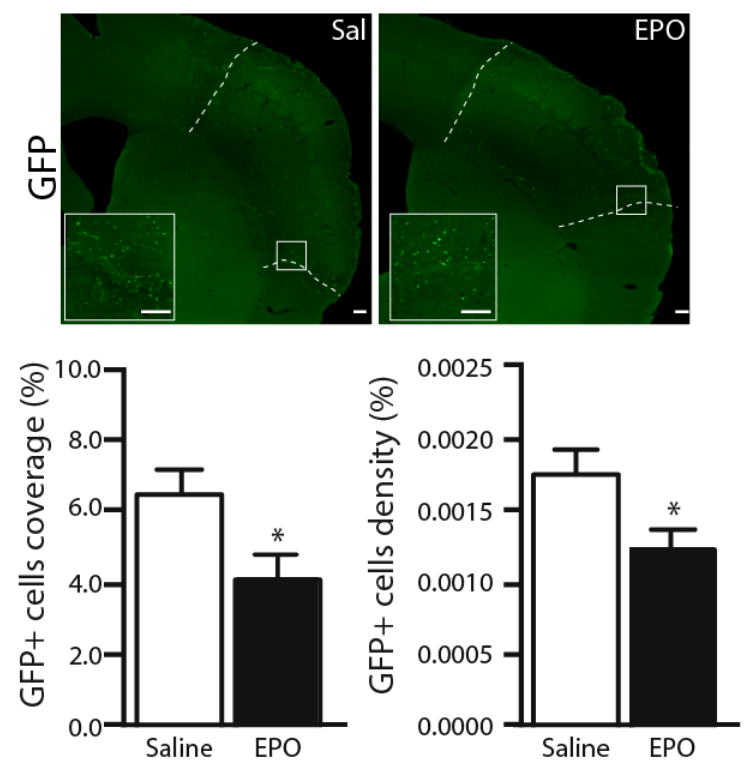

\section{0 months}

C
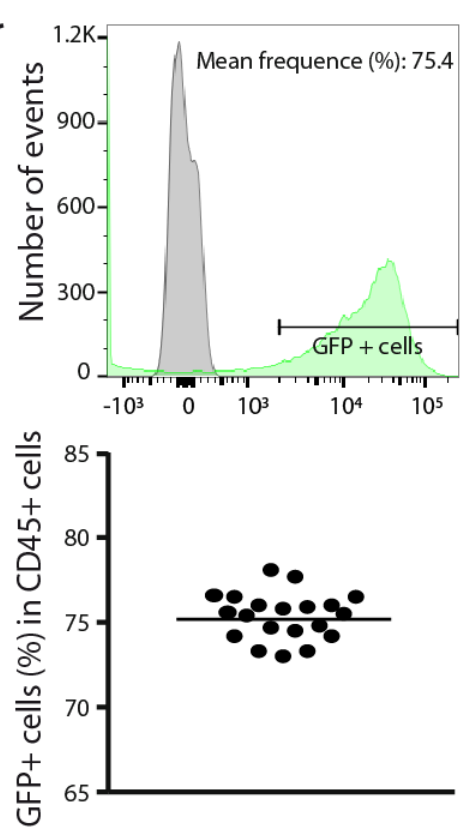
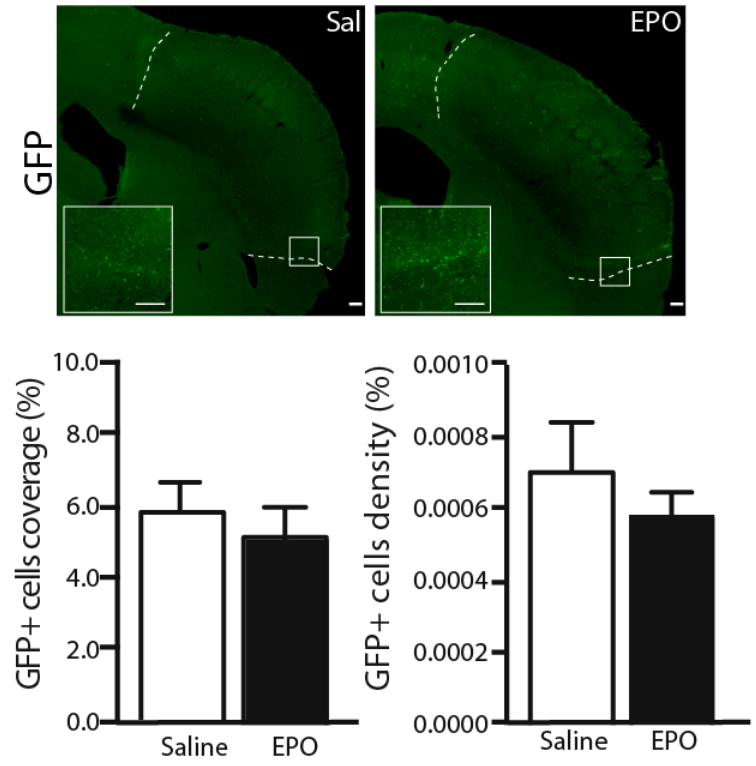

Figure 3: rhEPO limits brain infiltration of BMDC in the ischemic injury. Representative histograms overlay (A. C. upper panel) showing the gating strategy for GFP-positive cells (green) relatively to GFP-negative cells derived from WT controls (grey). Representative graphs (A, C lower panel) showing frequencies of GFP-positive cells (relative \% of CD45-positive cells) in 5- (A) and 20- (C) months old (GFP-WT) chimeric mice. Representative photomicrographs (B., D. upper panels) and respective stereological quantifications of GFP-positive cells coverage and density (relative \% of the total brain area) in saline- or EPO-treated 5- (B, lower panels) and 20- (D, lower panels) months old animals. Values are expressed as means \pm SEM. Statistical analyses were performed using an unpaired t test. $*=p<0.05$ significant difference compared to saline. $\mathrm{n}=8-9$ mice per group. Scale bars: $\mathrm{B}, \mathrm{D}=150 \mu \mathrm{m}$. Abbreviations: GFP: green fluorescent protein; EPO: erythropoietin; Sal: saline. 
patients may be potential candidates for rhEPO treatment, when not suitable for thrombolysis [35].

Our study revealed a neuroprotective role for EPO, through its anti-inflammatory properties, in the context of ischemic stroke induced by a platelet-rich thrombus. Although beneficial, animals' responsiveness to EPO treatment is likely age-dependent since its neuroprotective effects are abolished with age. Thus, it is crucial to consider ageing and comorbidities in order to adapt ischemic stroke treatment for each patient. Therefore, further preclinical studies aiming at developing new therapeutic strategies for ischemic stroke must consider age in animal models, in order to better translate experimental findings into clinics.
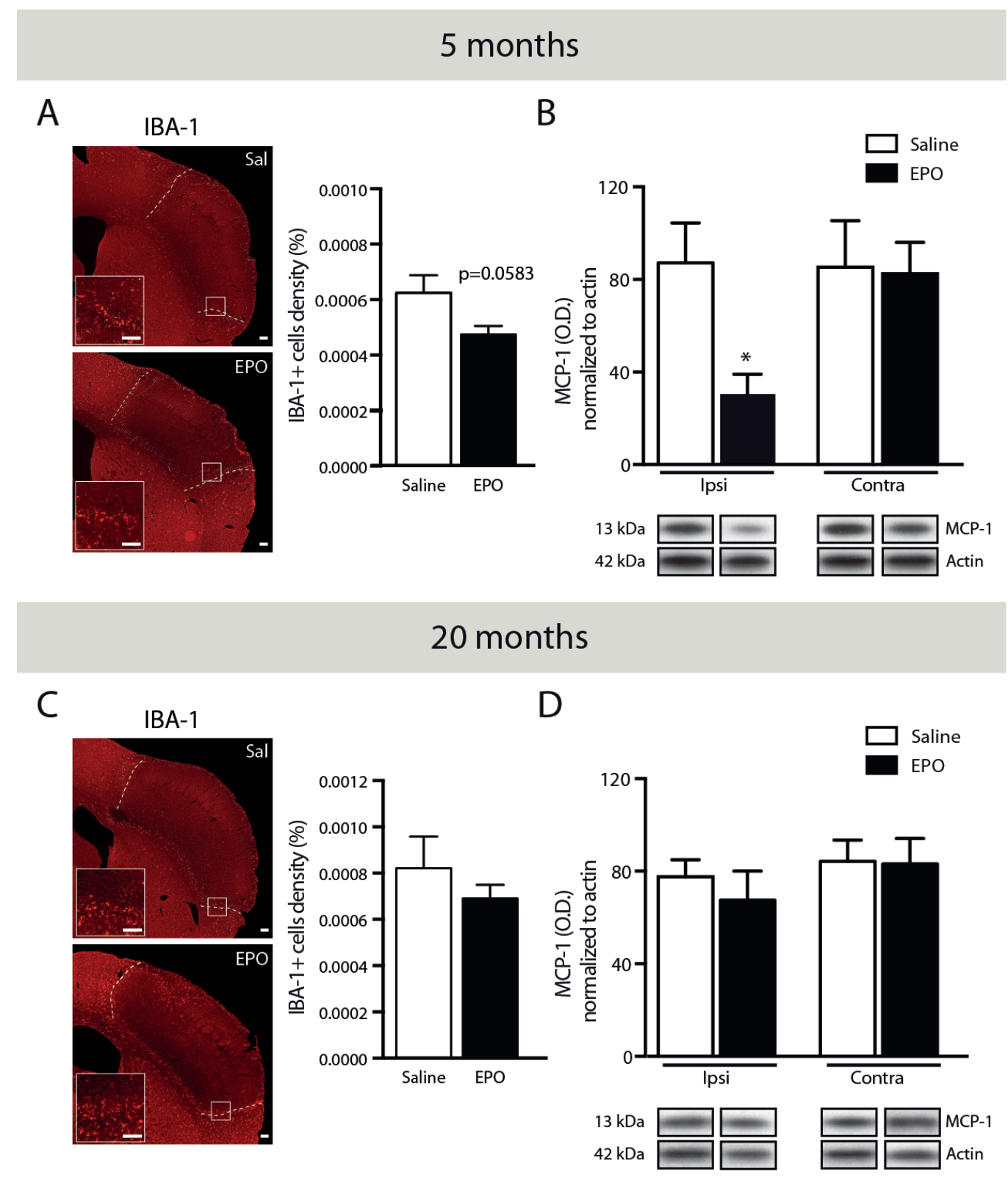

Figure 4: rhEPO administration limits microglial cell recruitment and reduces MCP-1 protein levels in ischemic injury. Representative photomicrographs of IBA-1 immunostaining and respective stereological quantification of microglial cells density (relative \% of the total brain area) of saline- or EPO-treated 5- A. and 20- C. months old animals. Quantifications by western blot analyses of brain MCP-1 protein levels normalized to $\beta$-actin protein levels in ischemic (ipsilateral hemisphere) in comparison to healthy tissue (contralateral hemisphere) of saline- or EPO-treated 5- B. and 20- D. months old animals. Represented bands are cropped blots derived from the same experiment. Values are expressed as mean \pm SEM. Statistical analyses were performed using an unpaired $t$-test. $*=p<0.05$ significant difference compared to saline. $n=8-9$ mice per group in $\mathrm{A}$ and $\mathrm{C}, n=4$ mice per group in $\mathrm{B}$ and $\mathrm{D}$. Scale bars: A, C = 150 $\mu \mathrm{m}$. Abbreviations: Contra: contralateral, EPO: erythropoietin; IBA-1: ionized calcium-binding adapter molecule 1; Ipsi: ipsilateral, MCP-1: monocyte chemotactic protein-1; OD: optical density; Sal: saline; WT: wild-type. 


\section{MATERIALS AND METHODS}

Complete details of materials and methods are described in online supplement as supplemental methods.

\section{Animals}

Experiments were performed according to the Canadian Council on Animal Care guidelines, as administered by the Laval University Animal Welfare Committee. Three- (i.e. young) and 18- (i.e. aged) months old adult male wild-type (WT; C57BL/6J) and agematched green fluorescent protein $\left[\mathrm{GFP}^{+/} ;\right.$; $57 \mathrm{BL} / 6-\mathrm{Tg}$ (CAG-EGFP) Osb/J)] mice were used.

\section{Chimeric mouse conditioning and BM transplantation}

WT chimeric mice were generated by transplanting $\mathrm{BM}$ cells isolated from $\mathrm{GFP}^{+/}$mice in irradiated WT mice $(\mathrm{GFP} \rightarrow \mathrm{WT})$, as previously described [37]. The chimerism level was assessed by flow cytometry (FACS) analysis 6 weeks after BM transplantation. Experimental protocol was initiated 2 weeks later.

\section{Experimental design}

Five- (i.e. young) and 20- (i.e. aged) months old $\mathrm{C} 57 / \mathrm{B} 16$ chimeric $(\mathrm{GFP} \rightarrow \mathrm{WT})$ mice were used (see timeline in Figure 1). Mice at 3 and 18 months of age were exposed to whole body irradiation prior to GFP-tagged BM-derived cells (BMDC) transplantation (-60 d) and chimerism was assessed (-14 d) by FACS analyses. Then, thrombosis-induced occlusion of the middle cerebral artery (MCAo) was induced by focal application of $30 \%$ ferric chloride $\left(\mathrm{FeCl}_{3}\right)$ solution, thus inducing ischemic injury $(0 \mathrm{~d})$. rhEPO administration was performed 24 hours following ischemic stroke and blood-circulating monocyte populations were assessed 3 hours post-injection $(1 \mathrm{~d})$. Finally, animals were sacrificed 24 hours post-treatment for immunostaining and biochemical analyses (2d).

\section{Focal cerebral ischemia and rhEPO administration}

Thrombus formation was induced by $30 \% \mathrm{FeCl}_{3}$ (Sigma-Aldrich) application on the middle cerebral artery (MCA) [38]. rhEPO (2500 IU/kg, EPREX ${ }^{\circledR} 4000$, Janssen) or vehicle (i.e. saline solution) was intraperitoneally injected 24 hours post-ischemic stroke.

\section{FACS analysis}

To study monocyte populations in the blood circulation, facial vein blood was collected 3 hours postrhEPO or saline administration. FACS analyses were performed as previously described [39].

\section{Tissue collection}

Animals were euthanized 24 hours post-rhEPO or saline administration (i.e. 48 hours post-ischemic stroke). Brains were removed, cut into $25 \mu \mathrm{m}$-thick coronal sections using a freezing microtome (Leica Microsystems), collected in anti-freeze solution prior to histological analyses.

\section{Fluoro-Jade B staining and immunofluorescence}

Neuronal death was assessed on coronal sections by Fluoro-Jade B (FJB) staining as described previously [40]. Microglial cells were detected by ionized calcium-binding adapter molecule-1 (IBA-1) immunofluorescent staining, as described previously [41]. Sections were mounted onto Micro Slides Superfrost ${ }^{\circledR}$ Plus and coverslipped with fluoromount G. Quantification of $\mathrm{FJB}^{+}$, IBA-1 $1^{+}, \mathrm{GFP}^{+}$ cellular density and coverage area were performed by unbiased stereological analyses [42].

\section{Histochemical immunostaining}

Blood-brain barrier (BBB) breakdown was assessed by immunoglobulins $\mathrm{G}$ ( $\operatorname{IgG}$ ) immunostaining ${ }^{31}$ and sections were digitized prior to quantification of $\mathrm{IgG}^{+}$ coverage area (i.e. extravasation) using ImageJ software.

\section{Western blot analysis}

Protein lysates were obtained from dissection of ischemic (ipsilateral hemisphere) and healthy (contralateral hemisphere) tissues and subjected to SDS-PAGE prior to western blot analysis. Anti-mouse monocyte chemotactic protein-1 (MCP-1) (1:1000; Cell Signaling) and anti-mouse $\beta$-actin (1:40000; EMD Millipore) primary antibodies were used prior to detection by horseradish peroxidase (HRP)-conjugated secondary antibodies and revelation by enhanced chemiluminescence plus (ECL) solution (GE Healthcare Life Sciences). Blots were digitized and proteins level were densitometrically analyzed with ImageJ software. 


\section{Statistical analysis and image preparation}

Results are expressed as mean \pm SEM. Statistical analyses were performed using standard two-tailed unpaired $t$-test for comparison between 2 groups (salineor rhEPO-treated groups), using GraphPad Prism software. All panels presented were assembles using Adobe Photoshop CS5 (version 12.0.4) and Adobe Illustrator CS5 (version 15.0.2).

\section{ACKNOWLEDGMENTS}

We thank Dr. Giulia Cisbani, Mrs. Marie-Michèle Plante and Mrs. Nataly Laflamme for image preparation and technical support.

\section{CONFLICTS OF INTEREST}

There is no conflict of interest.

\section{FUNDING}

This work was supported by the Canadian Institutes in Health Research (CIHR). PT is supported by the Canadian Institutes in Health Research (CIHR). ALB is supported by the Fonds de la Recherche Québec Santé (FRQS). AEA is supported by the CRCHU de Québec Research Center - Université Laval. SR is supported by a Canadian Research Chair in Neuroimmunology.

\section{REFERENCES}

1. Lloyd-Jones D, Adams RJ, Brown TM, Carnethon M, Dai S, De Simone G, Ferguson TB, Ford E, Furie K, Gillespie C, Go A, Greenlund K, Haase N, et al. Executive summary: heart disease and stroke statistics-2010 update: a report from the American Heart Association. Circulation. 2010;121:948-954.

2. Gorelick PB, Wong KS, Bae H-J, Pandey DK. Large artery intracranial occlusive disease: a large worldwide burden but a relatively neglected frontier. Stroke. 2008;39:2396-2399.

3. Falati S, Gross P, Merrill-Skoloff G, Furie BC, Furie B. Real-time in vivo imaging of platelets, tissue factor and fibrin during arterial thrombus formation in the mouse. Nat. Med. 2002;8:1175-1181.

4. Roussel BD, Macrez R, Jullienne A, Agin V, Maubert E, Dauphinot L, Potier MC, Plawinski L, Castel H, Hommet Y, Munuera J, Montaner J, Yepes M, et al. Age and albumin $\mathrm{D}$ site-binding protein control tissue plasminogen activator levels: neurotoxic impact. Brain. 2009;132:2219-2230.

5. Wiman B, Collen D. Molecular mechanism of physiological fibrinolysis. Nature. 1978;272:549-550.

6. Young AR, Ali C, Duretête A, Vivien D. Neuroprotection and stroke: time for a compromise. J. Neurochem.
2007;103:1302-1309.

7. Vivien D, Gauberti M, Montagne A, Defer G, Touzé E. Impact of tissue plasminogen activator on the neurovascular unit: from clinical data to experimental evidence. J. Cereb. Blood Flow Metab. 2011;31:2119-2134.

8. The National Institute of Neurological Disorders (NIND) and Stroke rt-PA Stroke Study Group. Tissue plasminogen activator for acute ischemic stroke. N. Engl. J. Med. 1995;333:1581-1587.

9. Jang IK, Gold HK, Ziskind AA, Fallon JT, Holt RE, Leinbach RC, May JW, Collen D. Differential sensitivity of erythrocyte-rich and platelet-rich arterial thrombi to lysis with recombinant tissue-type plasminogen activator. A possible explanation for resistance to coronary thrombolysis. Circulation. 1989;79:920-928.

10. Kim EY, Heo JH, Lee S-K, Kim DJ, Suh S-H, Kim J, Kim DI. Prediction of thrombolytic efficacy in acute ischemic stroke using thin-section noncontrast CT. Neurology. 2006;67:1846-1848.

11. Hacke W, Kaste M, Bluhmki E, Brozman M, Dávalos A, Guidetti D, Larrue V, Lees KR, Medeghri Z, Machnig $\mathrm{T}$, Schneider D, von Kummer R, Wahlgren N, et al. Thrombolysis with alteplase 3 to 4.5 hours after acute ischemic stroke. N. Engl. J. Med. 2008;359:1317-1329.

12. Lees KR, Bluhmki E, von Kummer R, Brott TG, Toni D, Grotta JC, Albers GW, Kaste M, Marler JR, Hamilton SA, Tilley BC, Davis SM, et al. Time to treatment with intravenous alteplase and outcome in stroke: an updated pooled analysis of ECASS, ATLANTIS, NINDS, and EPITHET trials. Lancet. 2010;375:1695-1703.

13. Reeves MJ, Arora S, Broderick JP, Frankel M, Heinrich JP, Hickenbottom S, Karp H, LaBresh KA, Malarcher A, Mensah G, Moomaw CJ, Schwamm L, Weiss P, et al. Acute stroke care in the US: results from 4 pilot prototypes of the Paul Coverdell National Acute Stroke Registry. Stroke. 2005;36:1232-1240.

14. Jelkmann W. Erythropoietin: structure, control of production, and function. Physiol. Rev. 1992;72:449-489.

15. Grover A, Mancini E, Moore S, Mead AJ, Atkinson D, Rasmussen KD, O'Carroll D, Jacobsen SE, Nerlov C. Erythropoietin guides multipotent hematopoietic progenitor cells toward an erythroid fate. J. Exp. Med. 2014;211:181188 .

16. Brines M, Cerami A. Discovering erythropoietin's extrahematopoietic functions: biology and clinical promise. Kidney Int. 2006;70:246-250.

17. Sirén AL, Radyushkin K, Boretius S, Kämmer D, Riechers CC, Natt O, Sargin D, Watanabe T, Sperling S, Michaelis T, Price J, Meyer B, Frahm J, et al. Global brain atrophy after unilateral parietal lesion and its prevention by erythropoietin. Brain. 2006;129:480-489.

18. Wang L, Zhang Z, Wang Y, Zhang R, Chopp M. Treatment of stroke with erythropoietin enhances neurogenesis and angiogenesis and improves neurological function in rats. 
Stroke. 2004:35:1732-1737.

19. Bernaudin M, Marti HH, Roussel S, Divoux D, Nouvelot A, MacKenzie, Petit E. A potential role for erythropoietin in focal permanent cerebral ischemia in mice. J. Cereb. Blood Flow Metab. 1999;19:643-651.

20. Zhang L, Zhang ZG, Chopp M. The neurovascular unit and combination treatment strategies for stroke. Trends Pharmacol. Sci. 2012;33:415-422.

21. Leist M, Ghezzi P, Grasso G, Bianchi R, Villa P. Derivatives of erythropoietin that are tissue protective but not erythropoietic. Science 2004;305:239-242.

22. Iwai M, Cao G, Yin W, Stetler RA, Liu J, Chen J. Erythropoietin promotes neuronal replacement through revascularization and neurogenesis after neonatal hypoxia/ ischemia in rats. Stroke. 2007;38:2795-2803.

23. Souvenir R, Doycheva D, Zhang JH, Tang J. Erythropoietin in stroke therapy: friend or foe. Curr. Med. Chem. 2015;22:1205-1213.

24. Shapira S, Sapir M, Wengier A, Grauer E, Kadar T. Aging has a complex effect on a rat model of ischemic stroke. Brain Res.. 2002;925:148-158.

25. Li Y, Lu Z, Keogh CL, Yu SP, Wei L. Erythropoietininduced neurovascular protection, angiogenesis, and cerebral blood flow restoration after focal ischemia in mice. J. Cereb. Blood Flow Metab. 2007;27:1043-1054.

26. Wang R, Wu X, Liang J, Qi Z, Liu X, Min L, Ji X, Luo $\mathrm{Y}$, Zhao H. Intra-artery infusion of recombinant human erythropoietin reduces blood-brain barrier disruption in rats following cerebral ischemia and reperfusion. Int. J. Neurosci. 2015;125:693-702.

27. Le Behot A, Gauberti M, De Lizarrondo SM. GpIb $\alpha$-VWF blockade restores vessel patency by dissolving platelet aggregates formed under very high shear rate in mice. Blood. 2014;123;3354-3363.

28. Geissmann F, Jung S, Littman DR. Blood monocytes consist of two principal subsets with distinct migratory properties. Immunity. 2003;19:71-82.

29. Naert G, Rivest S. A deficiency in CCR2+ monocytes: the hidden side of Alzheimer's disease. J. Mol. Cell Biol. 2013;5:284-293.

30. Diaz-Otero JM, Garver H, Fink GD, Jackson WF, Dorrance AM. Aging is associated with changes to the biomechanical properties of the posterior cerebral artery and parenchymal arterioles. Am. J. Physiol. Heart Circ. Physiol. 2016;310:H365-75.

31. Fotheringham AP, Davies CA, Davies I. Oedema and glial cell involvement in the aged mouse brain after permanent focal ischaemia. Neuropathol. Appl. Neurobiol. 2000;26:412-423.

32. Lucke-Wold BP, Logsdon AF, Turner RC, Rosen CL, Huber JD. Aging, the metabolic syndrome, and ischemic stroke: redefining the approach for studying the blood-brain barrier in a complex neurological disease. Adv. Pharmacol. 2014;71:411-449.
33. Villa P, Bigini P, Mennini T, Agnello D, Laragione T, Cagnotto A, Viviani B, Marinovich M, Cerami A, Coleman TR, Brines M, Ghezzi P. Erythropoietin selectively attenuates cytokine production and inflammation in cerebral ischemia by targeting neuronal apoptosis. J. Exp. Med. 2003;198:971-975.

34. Ehrenreich H, Hasselblatt M, Dembowski C, Cepek L, Lewczuk P, Stiefel M, Rustenbeck HH, Breiter N, Jacob S, Knerlich F, Bohn M, Poser W, Rüther E, et al. Erythropoietin therapy for acute stroke is both safe and beneficial. Mol. Med. 2002;8:495-505.

35. Tsai TH, Lu CH, Wallace CG, Chang WN, Chen SF, Huang $\mathrm{CR}$, et al. Erythropoietin improves long-term neurological outcome in acute ischemic stroke patients: a randomized, prospective, placebo-controlled clinical trial. Crit. Care. 2015;19:49.

36. Zechariah A, ElAli A, Hermann DM. Combination of Tissue-Plasminogen Activator With Erythropoietin Induces Blood-Brain Barrier Permeability, Extracellular Matrix Disaggregation, and DNA Fragmentation After Focal Cerebral Ischemia in Mice. Stroke. 2010;41:1008-1012.

37. Lampron A, Lessard M, Rivest S. Effects of Myeloablation, Peripheral Chimerism and Whole Body Irradiation on the Entry of Bone Marrow-Derived Cells Into the Brain. Cell Transplant. 2011;21;1149-1159.

38. Karatas H, Erdener SE, Gursoy-Ozdemir Y, Gurer G, Soylemezoglu F, Dunn AK, Dalkara T. Thrombotic distal middle cerebral artery occlusion produced by topical $\mathrm{FeCl}(3)$ application: a novel model suitable for intravital microscopy and thrombolysis studies. J. Cereb. Blood Flow Metab. 2011;31:1452-1460.

39. Lampron A, Pimentel-Coelho PM, Rivest S. Migration of bone marrow-derived cells into the central nervous system in models of neurodegeneration. J. Comp. Neurol. 2013;521:3863-3876.

40. Turrin NP, Rivest S. Molecular and Cellular Immune Mediators of Neuroprotection. Mol. Neurobiol. 2006;34:221-242.

41. ElAli A, Bordeleau M, Thériault P, Filali M, Lampron A, Rivest S. Tissue-Plasminogen Activator Attenuates Alzheimer's Disease-Related Pathology Development in APPswe/PS1 Mice. Neuropsychopharmacology. 2015;41:1297-1302.

42. Boissonneault V, Filali M, Lessard M, Relton J, Wong G, Rivest S. Powerful beneficial effects of macrophage colonystimulating factor on beta-amyloid deposition and cognitive impairment in Alzheimer's disease. Brain. 2009;132:10781092. 